\title{
Experiencing CSCL: from motivation to the embodied experience and beyond
}

\author{
Carolyn Rosé $^{1} \cdot$ Sanna Järvelä ${ }^{2} \cdot$ Sten Ludvigsen ${ }^{3}$ \\ Published online: 24 March 2020 \\ (C) International Society of the Learning Sciences, Inc. 2020
}

\section{Introduction}

This March edition of the International Journal of Computer-Supported Collaborative Learning marks a transition into a new phase in the life of the journal, and thus the former and new editors-in-chief are joining together for this transitional editorial. Here we take the opportunity to highlight the work coming out in this issue as well as to lay out a vision for this new era.

In order to stay current and remain on the forefront of research it will be our goal to keep our eyes open to emerging technologies with potential to enhance the ability to model collaborative processes both for assessment of processes and for triggering real time support for those processes, and to offer a wide range of rich instructional experiences. This includes embracing emerging opportunities with big data and emerging modeling technologies. But these opportunities must be paired with deep theories and conceptual models of collaboration and learning than enable formation of research questions and hypotheses, and ultimately the accumulation of knowledge. To keep informing the field with evidence based solutions we highlight strong empirical CSCL research targeting process improvements and learning gains that new technologies can contribute to the field of collaborative learning.

In line with our vision, we bring four articles to this issue, first one that offers a backwards glance of a sort, specifically a meta-analysis of research in the area of script-based collaboration, reconsidering questions that have often been pondered, now with evidence to substantiate specific claims, some of which are contrary to expectation. We then transition to a more forward glance with three papers highlighting recent work continuing the trend to bring collaborative learning out of the screen and into the face-to-face world (Tissenbaum et al. 2017). Specifically, these three papers represent the emerging area of the embodied experience in CSCL, offering theoretical foundations, new measurement technology, and innovative experiences for students.

Carolyn Rosé

cp3a@andrew.cmu.edu

1 Carnegie Mellon University, Pittsburgh, PA, USA

2 University of Oulu, Oulu, Finland

3 University of Oslo, Oslo, Norway 


\section{A backwards glance reveals and unexpected positive effect}

The first paper, by Anika Radkowitsch, Freydis Vogel, and Frank Fischer, "Good for learning, bad for motivation? A Meta-Analysis on the Effects of Computer-Supported Collaboration Scripts on Learning and Motivation" offers a meta-analysis of literature in the area of scripted collaboration, in part specifically investigating what evidence exists that experiencing external scripts for structuring collaboration may result in reduced motivation.

Their meta-analysis stimulates important discussion in the journal: What are the very core components in human learning? How they are evidenced in CSCL? Motivation is a driving force in human learning and different motivational constructs interact in collaborative learning engagement. The meta-analysis synthesizes the results of 53 primary studies that experimentally compared the effect of learning with a CSCL script to unguided collaborative learning on at least one of the key outcome variables of motivation, domain learning, and collaboration process skills. Even though their analysis shows that learning with CSCL scripts leads to a non-significant positive effect on motivation, it offers important counter evidence against the criticism that CSCL scripts have negative motivational effects. The findings point to positive effects on domain learning and collaboration skills. Interestingly, the meta-analysis shows how scaffolding single particular collaborative activities and scaffolding a combination of collaborative activities affects the impact of CSCL scripts. The meta-analysis encourages the field to continue discussing various concepts and components of collaborative learning processes and how they can best be supported.

\section{A forward look towards new experiences}

The next two papers focus on embodiment and CSCL. The first such article, by Joshua Danish, Noel Eynedy, Asmalina Saleh, and Megan Humburg, "Learning in Embodied Activity Framework: A Sociocultural Framework for Embodied Cognition" offers a recasting of Activity Theory for Embodied CSCL experiences. It makes a case for why it is valuable to think explicitly about embodiment in the context of Activity Theory, especially as it is challenging to account for both individual and group level phenomena within the same framework. One valuable contribution of the article is that it offers a rich exposition of observations from several studies where this new lens was used to characterize how embodied science learning plays out in mixed reality experiences in naturalistic settings such as the classroom.

The next article, by Bertrand Schneider, Yong Dich, and Iulian Radu, "Unpacking the Relationship Between Existing and New Measures of Physiological Synchrony and Collaborative Learning: A Mixed Methods Study", continues on the theme of embodiment by providing a new suite of measurement instruments for studying embodied CSCL through physiological sensors. This article breaks new ground in exploring how physiological synchrony relates to collaborative processes.

The paper also continues the discussion we have started in the journal earlier: How physiological measures can contribute to understanding about productive collaborative learning interactions and how physiological synchrony relates to collaborative learning (e.g. Malmberg et al. 2019). Schneiner and colleagues' data come from 42 pairs of participants $(N=84)$ who worked on programming a robot to solve a variety of mazes. Their findings show partly contradictory and partly complementary findings as compared to prior studies. The 
message is that careful attention is needed for operationalizing constructs measured with physiological data evidencing the role of physiological synchrony in collaborative learning. Perhaps physiological measures offer a new means to complement other existing CSCL research methods, such as video observations, as suggested in earlier articles (Järvelä et al. 2019). Beyond its in depth quantitative analysis, the article provides a qualitative analysis highlighting important differences between a low performing and a high performing group, which illustrates ways in which it might be possible to pinpoint productive and unproductive collaboration episodes through physiological data. These measures are still very new in the CSCL research and the paper brings important new information pointing towards future opportunities for innovative collaboration support, but also challenges for measuring and analysing collaborative processes, which suggests room for methodological development work. This work takes the field one step further in its progress towards multi-modal understanding of collaboration, synergizing with Schneider's recent work related to eye tracking (Schneider et al. 2018; Schneider 2019).

Continuing investigations of designing authentic learning experiences for science classrooms (Hod and Sagy 2019), the final article of this issue, by Yotam Hod and Daniel Twersky, "Distributed Spatial Sensemaking on the Augmented Reality Sandbox" explores a novel environment for embodied CSCL experiences in an environment referred to as the Augmented Reality Sandbox. In contrast to prior research, which has focused on AR Sandbox activity designs, outcome measures, and user's perceptions of different usability functions, this article probes deeply into the situated processes by which groups engage in CSCL activities. Within this Augmented Reality setting, the article applies earlier work related to distributed spatial sensemaking in order to analyze group interactions spanning material, social, and activity contexts.

\section{A word from the new co-editors-in-chief}

As new co-editors-in-chief, we thank our predecessor Sten. He has served this journal faithfully over the past four years. He stepped into the difficult position of taking over the journal from its founder. And he did it with grace and hard work.

Moving into a new era, our vision is to build on our complementary expertise within the CSCL community, in order to reach a more diverse readership and authorship community, and thus achieve greater momentum for pushing the research field forward. Building on Carolyn' editorial experience with the past Multivocality effort published within the CSCL book series, we will take an inclusive stance within the community in order to embrace a diversity of innovative technologies and research methodologies, while in all ways striving for rigor appropriate to the chosen methods for each accepted paper. Within this rich and broad theoretical and methodological structure, we will seek contributions that bring CSCL to new contexts, with an eye towards workplace learning, learning in online communities, personal development and career advancement, and communities of practice.

Our expertise lies in this exciting intersection: Carolyn's expertise is grounded in Artificial Intelligence, Machine Learning, Computational Linguistics, and the Learning Sciences. Her specific expertise in computer-supported collaborative learning includes automated collaborative process analysis, dynamic support for collaborative learning, computational sociolinguistics, and other quantitative methods including machine learning. Sanna's background is in educational psychology, learning sciences and educational technology. Her focus is on 
understanding learning processes, such as motivation, affect, cognition and metacognition in solo and collaborative learning. She has experience in social, socioemotional and contextual aspects in collaborative learning. In her effort to understand cognitive, motivational and affective challenges in collaborative learning she has worked with self-regulated learning (SRL) theory and extended the theoretical and methodological means to understand regulation in collaborative learning, socially shared regulation (SSRL) in specific. Her research covers applying technology for supporting learning processes and developing practical models for CSCL. Sanna has been working with various process oriented, mixed methods and multimodal methods (video, physiological data, logdata, situated self-reports etc.). Having her background understanding complex processes in collaborative learning we are also seeking for contributions that will push the theoretical and conceptual discussion on collaborative learning process forward and contribute to empirical research and theory building with new methodological means.

As we move into this new era of journal leadership, our aim will be to continually strive for increasing scientific evidence for the accumulation of scientific knowledge in the field in our role facilitating the flow of top quality empirical research papers into this esteemed journal. Strong empirical evidence needed in order to achieve success, and thus we will seek high quality empirical studies with strong research designs grounded in rich theoretical models. In all of this, we will strive to build on our history while striving for the future. For example, strong and valid "traditional" CSCL research designs and methods are important, but also new and renewed methodological approaches that have the potential to increase the evidence base in CSCL. We will work for increasing the journal efficiency and visibility and more intensive engagement within and across journal communities.

\section{References}

Hod, Y., \& Sagy, O. (2019). Conceptualizing the designs of authentic computer-supported collaborative learning environments in schools. International Journal of Computer-Supported Collaborative Learning, 14(2), 143164.

Järvelä, S., Järvenoja, H., \& Malmberg, J. (2019). Capturing the dynamic and cyclical nature of regulation: Methodological progress in understanding socially shared regulation in learning. International Journal of Computer Supported Collaborative Learning, 4, 425-441. https://doi.org/10.1007/s11412-019-09313-2.

Malmberg, J., Haataja, E., Seppänen, T., \& Järvelä, S. (2019). Are we together or not? The temporal interplay of monitoring, physiological arousal and physiological synchrony during a collaborative exam. International Journal of Computer-Supported Collaborative Learning, 14(4), 467-490.

Schneider, B., Sharma, K., Cuendet, S., Zufferey, G., Dillenbourg, P., \& Pea, R. (2018). Leveraging mobile eyetrackers to capture joint visual attention in co-located collaborative learning groups. International Journal of Computer-Supported Collaborative Learning, 13(3), 241-261.

Schneider, B. (2019). Unpacking collaborative learning processes during hands-on activities using Mobile eyetracking. Proceedings of 13 th international conference of computer supported collaborative learning, 156165 .

Tissenbaum, M., Berland, M., \& Lyons, L. (2017). DCLM framework: Understanding collaboration in openended tabletop learning environments. International Journal of Computer-Supported Collaborative Learning, 12(1), 35-64.

Publisher's note Springer Nature remains neutral with regard to jurisdictional claims in published maps and institutional affiliations. 